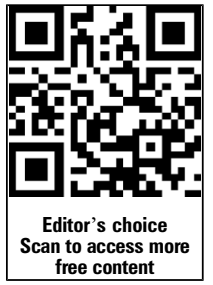

an to access mor free content

${ }^{1}$ Motor Behavior Laboratory, University of Florida, Gainesville, Florida, USA ${ }^{2}$ Human Motor Control Laboratory, University of Tasmania, Hobart, Tasmania, Australia

${ }^{3}$ Research Institute for Sport and Exercise Sciences, Liverpool John Moores University, Liverpool, UK

\section{Correspondence to} Dr James H Cauraugh, Motor Behavior Laboratory, Department of Applied Physiology and Kinesiology, University of Florida, Gainesville, FL 32611-8206, USA; cauraugh@ufl.edu

Received 12 May 2015 Revised 6 August 2015 Accepted 10 August 2015 Published Online First 28 August 2015

\section{SLinked}

- http://dx.doi.org/10.1136/ jnnp-2015-311991

\section{CrossMark}

\section{To cite: Kang $N$}

Summers JJ, Cauraugh JH. J Neurol Neurosurg Psychiatry 2016:87:345-355

\title{
Transcranial direct current stimulation facilitates motor learning post-stroke: a systematic review and meta-analysis
}

\author{
Nyeonju Kang, ${ }^{1}$ Jeffery J Summers, ${ }^{2,3}$ James H Cauraugh ${ }^{1}$
}

\begin{abstract}
Transcranial direct current stimulation (tDCS) is an attractive protocol for stroke motor recovery. The current systematic review and meta-analysis investigated the effects of tDCS on motor learning post-stroke. Specifically, we determined long-term learning effects by examining motor improvements from baseline to at least 5 days after tDCS intervention and motor practise. 17 studies reported long-term retention testing (mean retention interval $=43.8$ days; $S D=56.6$ days) and qualified for inclusion in our meta-analysis. Assessing primary outcome measures for groups that received tDCS and motor practise versus sham control groups created 21 valid comparisons: (1) 16 clinical assessments and (2) 5 motor skill acquisition tests. A random effects model meta-analysis showed a significant overall effect size $=0.59\left(p<0.0001\right.$; low heterogeneity, $T^{2}=0.04$; $\mathrm{I}^{2}=22.75 \%$; and high classic fail-safe $\mathrm{N}=240$ ). 4 moderator variable analyses revealed beneficial effects of tDCS on long-term motor learning: (1) stimulation protocols: anodal on the ipsilesional hemisphere, cathodal on the contralesional hemisphere, or bilateral; (2) recovery stage: subacute or chronic stroke; (3) stimulation timing: tDCS before or during motor practise; and (4) task-specific training or conventional rehabilitation protocols. This robust meta-analysis identified novel long-term motor learning effects with tDCS and motor practise post-stroke.
\end{abstract}

\section{INTRODUCTION}

Stroke is a leading cause of chronic motor disabilities in the USA, and most likely worldwide. Over $50 \%$ of patients with chronic stroke display residual motor dysfunctions and hemiparesis. ${ }^{1}$ Unfortunately, stroke rehabilitation programmes have not solved the issues of motor dysfunctions and hemiparesis. ${ }^{1} 2$ Thus, the search for effective treatment protocols continues. An additional unanswered question concerns whether individuals who experienced a stroke learn and retain voluntary movements practised during therapy sessions. Determining long-term motor learning beyond a short acquisition phase will contribute to the development of training protocols to enhance motor recovery following stroke.

A current popular stroke treatment protocol is transcranial direct current stimulation (tDCS), a non-invasive brain stimulation technique. tDCS is an economical, portable and easily accessible protocol. Brain activity patterns change as weak direct currents (eg, 1-2 mA) are delivered to the scalp through sponge electrodes. For the past decade, tDCS studies reported that anodal tDCS stimulation typically increases cortical excitability whereas cathodal tDCS stimulation decreases cortical excitability in an animal model ${ }^{3} 4$ and in humans. ${ }^{5} 6$

Even though the mechanisms underlying tDCS are still unclear, the high number of studies reporting results from stimulation protocols demands a close examination of stroke rehabilitation. Indeed, the evidence from a systematic review that tDCS transiently improves motor performance poststroke $^{7}$ is unclear and whether long-term improvements in recovered movements are sustained is seldom discussed. ${ }^{7}{ }^{8}$ Two recent meta-analyses that did examine long-term motor learning after tDCS were unable to resolve the controversy perhaps because of the excessive heterogeneity in the few studies analysed and the potential confound by comparing studies that provided tDCS before motor training to studies that stimulated during motor training. ${ }^{10}$ The current systematic review and meta-analysis accommodates for the above limitations by comparing studies that investigated the effects of tDCS and motor practise training on long-term motor learning post-stroke.

The classic definition of motor learning as a set of internal processes facilitated by practise leading to relatively long-term changes in the capabilities to produce voluntary movements includes two distinct phases: (1) acquisition and (2) retention. ${ }^{11-13}$ The acquisition phase involves temporary changes in behaviour after practise whereas retention represents relatively long-term changes in goal-directed movements or motor skill learning. Comparing baseline motor performances with long-term retention testing provides motor learning evidence. ${ }^{12} 14$ Reacquiring voluntary movements post-stroke involves early and later motor learning stages as individual's practise initiating, controlling and terminating movements. Closely aligned with the practise component of motor skill learning is usedependent learning. Movement experiences increase the probability that motor skill learning will accrue. A contrary control approach is adaptive learning as described by Bastian ${ }^{15}$ in their recent split-belt treadmill studies. ${ }^{16}$

Further, we compared motor learning findings following tDCS intervention combined with a taskspecific training (eg, motor skill learning) to tDCS with conventional rehabilitation protocols (eg, constraint-induced movement therapy (CIMT); occupational or physical therapy). Rehabilitation 
specialists often structure therapy sessions that emphasise practising-specific voluntary/goal-directed movements or skills. This type of practise refers to task-specific training. On the other hand, for conventional therapy regardless of the type of rehabilitation practise, therapists monitor and evaluate motor capabilities across time to determine motor learning progress, plateaus and improvements. Given that, Winstein et $a l^{17}$ reported that task-specific training revealed more improved motor functions than standard care (eg, occupational therapy); the long-term motor learning effects of tDCS interventions combined with task-specific training may be greater than tDCS protocols with conventional therapy.

The current stroke meta-analysis is unique in two aspects: (1) long-term motor learning effects of tDCS combined with motor training in comparison to sham tDCS with motor training and (2) substantially more studies $(\mathrm{N}=17)$ were identified and submitted to the meta-analysis than previous reviews. ${ }^{9}{ }^{10}$ Further, we asked four leading questions: (1) Do tDCS protocols, anodal stimulation on ipsilesional hemisphere, cathodal stimulation on contralesional hemisphere or bilateral stimulation improve motor learning post-stroke? (2) Are sustained behavioural effects of tDCS interventions found in each stage of stroke recovery (ie, acute, subacute and chronic)? (3) Does timing of a tDCS intervention (ie, before vs during motor training) influence long-term motor learning in stroke survivors? Moreover, (4) Does tDCS combined with task-specific motor training versus conventional rehabilitation distinguish long-term motor improvement post-stroke?

\section{METHODS}

Literature search and study selection

Our literature search concentrated on tDCS studies that investigated long-term effects on motor functions post-stroke. We began searching in November 2014 and ended 30 June 2015. Publication dates ranged from 2005 to June 2015. An initial search included PubMed, ISI's Web of Knowledge and Cochrane Database of Systematic Reviews. Seven keywords were: (1) stroke, (2) cerebrovascular accident, (3) brain infarct, (4) transcranial direct current stimulation (tDCS), (5) motor learning, (6) long-term retention test (delayed) and (7) transfer task. We initially identified 55 potential research studies.

Inclusion criteria for our meta-analysis were: (1) quantitative evaluation of tDCS effects on motor learning post-stroke, (2) a retention interval at least 5 days postintervention, (3) tDCS stimulation (eg, anodal, cathodal or bilateral stimulations) comparing pretreatment and post-treatment, (4) active stimulation versus sham control comparison and (5) tDCS combined with motor training (eg, stimulation before motor training or stimulation during motor training). Following these criteria, 38 studies were excluded: (1) 12 review articles, (2) 15 studies with no retention testing, (3) 1 short retention interval, (4) 6 case studies: single participant or no statistical analyses, (5) 3 studies using tDCS protocols only and (6) 1 study that did not include a tDCS sham control group. Thus, the remaining 17 studies qualified for inclusion in our meta-analysis. ${ }^{18-35}$

Further, 1 of 17 qualified studies ${ }^{31}$ reported two retention tests: (1) retention after $24 \mathrm{~h}$ for 12 participants and (2) retention after 12 weeks for 5 participants. The first retention interval did not meet our inclusion criteria. The second retention interval did meet our inclusion criteria and we used the 12-week retention test values for our meta-analysis.

Thirteen of 17 studies reported one of three tDCS stimulation protocols (ie, anodal, cathodal, bilateral stimulation: anodal+ cathodal; $13 \times 1=13$ comparisons). Four studies reported both anodal and cathodal stimulation protocols (ie, $4 \times 2=8$ comparisons). Thus, 21 total comparisons from 17 studies were involved in our meta-analysis. All 21 comparisons compared active stimulation with sham control groups at the long-term retention test (mean retention interval $=43.8$ days; $\mathrm{SD}=56.6$ days).

Stimulation protocols were categorised with (1) anodal stimulation on M1 (primary motor cortex) of ipsilesional hemisphere (eight comparisons), (2) cathodal stimulation on S1M1 (primary sensorimotor cortex) of ipsilesional hemisphere (one comparison), (3) cathodal stimulation on M1 of contralesional hemisphere (seven comparisons) and (4) bilateral (anodal+cathodal) stimulation on both hemispheres (five comparisons). Studies for the three recovery stages post-stroke included: (1) acute (1 day1 month; two comparisons), (2) subacute (1-6 months; seven comparisons) and (3) chronic (greater than 6 months; 12 comparisons). ${ }^{36}$ Three stimulation protocols varied by onset in relation to motor training: (1) stimulation before motor training (8 comparisons) and (2) stimulation during motor training (13 comparisons). Sixteen comparisons used tDCS with conventional rehabilitation protocols (ie, CIMT, inpatient daily rehabilitation, occupational therapy and physical therapy) whereas five comparisons applied tDCS combined with taskspecific training (ie, motor skill learning and robot-assisted gait). Moreover, all 17 studies reported that participants signed an informed consent before beginning experimental protocols approved by ethics institutions. Table 1 displays the characteristics of the 17 qualified studies we included in this meta-analysis.

\section{Motor outcome measures}

Quantifying the effects of tDCS on motor learning and longterm retention involved primary outcome measures reported by 17 individual studies. The 21 collected primary outcome measures were categorised according to: (1) clinical assessments (16 comparisons) and (2) motor skill acquisition tests (5 comparisons). The clinical assessments included: (1) Fugl-Meyer Assessment (upper limb motor function; higher score indicates motor improvement; seven comparisons), (2) Jebsen-Taylor Hand Function Test (measure of unilateral hand function; decreased time to complete each task denotes improved motor function; three comparisons), (3) National Institute of Health Stroke Scale (overall stroke impairment; lower score reveals better recovery post-stroke; three comparisons), (4) modified Ashworth scale (assessment for the affected wrist and elbow; lower score indicates no muscle tone; one comparison), (5) Nine Hole Peg Test (hand function test; reduced time to put pegs into the holes shows motor improvement; one comparison) and (6) range of motion joint tests (upper limb functions; range of motion in wrist extension, elbow extension and shoulder abduction; one comparison). Five comparisons reported a broad set of action tests as motor skill acquisition: (1) circuit game (accurately keep the cursor within the track; two comparisons), (2) sequencing task (sequential pressing of a 5 -element sequence on a 4-button electronic keyboard with paretic hand; one comparison), (3) key pressing task (number of correct key presses with 2nd-5th digit of paretic fingers over $30 \mathrm{~s}$; one comparison) and (4) 6 min walking test (distance with maximum walking speed for $6 \mathrm{~min}$; one comparison). Each study contributed data from one primary motor outcome measure. This conventional procedure minimises data biasing effects. ${ }^{37} 38$

\section{Data synthesis and analysis}

Three additional tables display specific details for each of the 21 comparisons. Table 2 summarises relevant characteristics, tDCS 
Table 1 Characteristics of each comparison included in the present meta-analysis (studies listed alphabetically)

\begin{tabular}{|c|c|c|c|c|c|c|c|c|}
\hline Study & Total N & $\begin{array}{l}\text { Age } \\
\text { (years) }\end{array}$ & Gender & $\begin{array}{l}\text { Rx onset } \\
\text { post-stroke (month) }\end{array}$ & $\begin{array}{l}\text { Stroke } \\
\text { type }\end{array}$ & $\begin{array}{l}\text { Affected } \\
\text { hemisphere }\end{array}$ & $\begin{array}{l}\text { Initial } \\
\text { impairment }\end{array}$ & Recovery stage \\
\hline Bolognini et $a l^{18}$ & 14 & 46.7 & $9 \mathrm{~F}, 5 \mathrm{M}$ & 35.2 & $12 \mathrm{I}, 2 \mathrm{H}$ & $8 \mathrm{~L}, 6 \mathrm{R}$ & 26.0/66 (FMA) & Chronic \\
\hline Celnik et a $\left.\right|^{19}$ & 9 & 55.3 & $4 \mathrm{~F}, 5 \mathrm{M}$ & 55.7 & 91 & $5 \mathrm{~L}, 4 \mathrm{R}$ & 62.0/66 (FMA) & Chronic \\
\hline Di Lazzaro et $a^{25}$ & 20 & 64.8 & $7 \mathrm{~F}, 13 \mathrm{M}$ & 0.1 & 201 & $12 \mathrm{~L}, 8 \mathrm{R}$ & 5.9/42 (NIHSS) & Acute \\
\hline Fusco et $a l^{20}$ & 11 & 58.4 & $6 \mathrm{~F}, 5 \mathrm{M}$ & 1.0 & 111 & $6 \mathrm{~L}, 5 \mathrm{R}$ & $24.7 / 66$ (FMA) & Subacute \\
\hline Geroin et $a l^{21}$ & 30 & 62.7 & $7 \mathrm{~F}, 23 \mathrm{M}$ & 26.4 & 301 & NA & $79.9 / 100$ (ESS) & Chronic \\
\hline Hesse et $a l^{22}$ & 96 & 65.0 & $37 \mathrm{~F}, 59 \mathrm{M}$ & 1.0 & $96 I$ & $51 \mathrm{~L}, 45 \mathrm{R}$ & 8.0/66 (FMA) & Subacute \\
\hline Khedr et $a l^{23}$ & 40 & 58.3 & $14 \mathrm{~F}, 26 \mathrm{M}$ & 1.0 & 401 & $18 \mathrm{~L}, 22 \mathrm{R}$ & $10.7 / 42$ (NIHSS) & Subacute \\
\hline Kim et $a l^{24}$ & 18 & 57.8 & $5 \mathrm{~F}, 13 \mathrm{M}$ & 1.0 & 181 & $9 \mathrm{~L}, 9 \mathrm{R}$ & $37.2 / 66$ (FMA) & Subacute \\
\hline Lefebvre et $a l^{26}$ & 18 & 61.0 & $6 \mathrm{~F}, 12 \mathrm{M}$ & 31.2 & $16 \mathrm{I}, 2 \mathrm{H}$ & $8 \mathrm{~L}, 10 \mathrm{R}$ & 7.1/25 (PPT) & Chronic \\
\hline Lefebvre et $a^{27}$ & 19 & 65.0 & $3 \mathrm{~F}, 16 \mathrm{M}$ & 62.4 & NA & $14 \mathrm{~L}, 5 \mathrm{R}$ & $7.4 / 25$ (PPT) & Chronic \\
\hline Lindenberg et $a^{28}$ & 20 & 58.8 & $5 \mathrm{~F}, 15 \mathrm{M}$ & 35.4 & 201 & $13 \mathrm{~L}, 7 \mathrm{R}$ & 39.0/66 (FMA) & Chronic \\
\hline Mortensen et $a l^{33}$ & 16 & 63.1 & $7 \mathrm{~F}, 9 \mathrm{M}$ & 30.2 & NA & $8 \mathrm{~L}, 8 \mathrm{R}$ & $15.5 / 25$ (SIS, hand) & Chronic \\
\hline Nair et al ${ }^{29}$ & 14 & 55.8 & $5 \mathrm{~F}, 9 \mathrm{M}$ & 30.5 & $14 I$ & $8 \mathrm{~L}, 6 \mathrm{R}$ & 30.5/66 (FMA) & Chronic \\
\hline Rocha et $\left.a\right|^{34}$ & 21 & 58.4 & $6 \mathrm{~F}, 15 \mathrm{M}$ & 29.4 & NA & $11 \mathrm{~L}, 10 \mathrm{R}$ & 49.1/66 (FMA) & Chronic \\
\hline Sattler et $a \beta^{32}$ & 20 & 65.2 & $6 \mathrm{~F}, 14 \mathrm{M}$ & 0.2 & 201 & NA & 48.0/66 (FMA) & Acute \\
\hline Wu et $a l^{30}$ & 90 & 47.6 & $21 \mathrm{~F}, 69 \mathrm{M}$ & 6.0 & $53 \mathrm{I}, 37 \mathrm{H}$ & $43 \mathrm{~L}, 47 \mathrm{R}$ & 10.0/66 (FMA) & Chronic \\
\hline Zimerman et al ${ }^{31}$ & 12 & 58.3 & $6 \mathrm{~F}, 6 \mathrm{M}$ & 30.0 & 121 & $5 \mathrm{~L}, 7 \mathrm{R}$ & 64.0/66 (FMA) & Chronic \\
\hline
\end{tabular}

ESS, European Stroke Scale; F, female; FMA, Fugl-Meyer Assessment (upper extremity); H, haemorrhagic; I, ischaemic; L, left; M, male; NA, not available; NIHSS, National Institutes of Health Stroke Scale; PPT, Purdue Pegboard Test; R, right; Rx, treatment group; SIS, Stroke Impact Scale V.3.0.

rehabilitation protocols and study designs. Table 3 provides tDCS parameters and table 4 displays summary statistics, outcome measures, individual weighted effect sizes (ES), calculated overall ES, Q statistic, $\mathrm{I}^{2}, \mathrm{~T}^{2}$ and classic fail-safe $\mathrm{N}$. According to Borenstein et al, ${ }^{39}$ a random effects model is appropriate when ES between studies are posited as different. Thus, we used a random effects model for calculating the overall ES and individual ES for each subgroup. ${ }^{40}$
Measuring heterogeneity and publication bias

Cochran's Q, Higgins and Green's I ${ }^{2}$ and $T^{2}$ (estimate of $\tau^{2}$ ) are tests for heterogeneity between the studies. Determining heterogeneity is vital for the meta-analytic technique. ${ }^{41}{ }^{42} \mathrm{I}^{2}$ represents heterogeneity as percentage values to assess evidence as different than a statistical chance occurrence. ${ }^{43}$ Higgins and Green $^{42}$ reported that greater than $50 \%$ of $\mathrm{I}^{2}$ indicates substantial heterogeneity (inconsistency). $\mathrm{T}^{2}$ is an estimate of variance

Table 2 tDCS rehabilitation protocols

\begin{tabular}{|c|c|c|c|c|}
\hline Study & Limb & Treatment & Session & Study design \\
\hline Bolognini et $a l^{18}$ & Upper & tDCS during CIMT & 10 sessions & Double blind; randomised*; parallel group; sham controlled \\
\hline Celnik et $a l^{19}$ & Upper & PNS+tDCS before motor skill learning & 1 session & Double blind; randomisedt; cross-over; sham controlled \\
\hline Di Lazzaro et $a l^{25}$ & Upper & tDCS during CIMT & 5 sessions & Double blind; randomised ${ }^{*}$; parallel group; sham controlled \\
\hline Fusco et $a l^{20}$ & Upper & tDCS before IDR & 10 sessions & Double blind; randomisedt; parallel group; sham controlled \\
\hline Geroin et $a l^{21}$ & Lower & tDCS during robot-assisted gait training & 10 sessions & Double blind; randomisedt; parallel group; sham controlled \\
\hline Hesse et $a l^{22}$ & Upper & tDCS during bilateral arm robot training & 30 sessions & Double blind; randomised ; parallel group; sham controlled \\
\hline Khedr et $a l^{23}$ & Upper & tDCS before IDR & 6 sessions & Double blind; randomised $\neq$; parallel group; sham controlled \\
\hline Kim et $a l^{24}$ & Upper & tDCS during OT & 10 sessions & Double blind; randomised ; parallel group; sham controlled \\
\hline Lefebvre et $a l^{26}$ & Upper & tDCS during motor skill learning & 1 session & Double blind; randomisedt; cross-over; sham controlled \\
\hline Lefebvre et $a l^{27}$ & Upper & tDCS during motor skill learning & 1 session & Double blind; randomised*; cross-over; sham controlled \\
\hline Lindenberg et $\left.a\right|^{28}$ & Upper & tDCS during $\mathrm{PT}+\mathrm{OT}$ & 5 sessions & Double blind; randomised§; parallel group; sham controlled \\
\hline Mortensen et $a l^{33}$ & Upper & tDCS during OT & 5 sessions & Double blind; randomised§; parallel group; sham controlled \\
\hline Nair et $a l^{29}$ & Upper & tDCS during OT & 5 sessions & Double blind; randomised ${ }^{*}$; parallel group; sham controlled \\
\hline Rocha et $a l^{34}$ & Upper & tDCS before $\mathrm{mCIMT}$ & 12 sessions & Double blind; randomised $¥ ;$ parallel group; sham controlled \\
\hline Sattler et $a^{32}$ & Upper & rPNS+tDCS before IDR & 5 sessions & Double blind; randomisedt; parallel group; sham controlled \\
\hline Wu et $a l^{30}$ & Upper & tDCS before PT & 20 sessions & Double blind; randomisedt; parallel group; sham controlled \\
\hline Zimerman et $a^{31}$ & Upper & tDCS during motor skill learning & 1 session & Double blind; randomisedt; cross-over; sham controlled \\
\hline \multicolumn{5}{|c|}{$\begin{array}{l}\text { Randomisation method: } \\
\text { *Not reported. } \\
\text { †Computer-generated randomisation list. } \\
\text { ¥Sealed envelope. } \\
\text { §Stratified block randomisation. } \\
\text { CIMT, constraint-induced movement therapy; IDR, inpatient daily rehabilitation; mCIMT, modified constraint-induced movement therapy; OT, occupational therapy; PNS, peripheral nerve }\end{array}$} \\
\hline
\end{tabular}


Table 3 tDCS parameters

\begin{tabular}{|c|c|c|c|c|c|c|c|c|c|}
\hline \multirow[b]{2}{*}{ Study } & \multicolumn{4}{|c|}{ Stimulation } & \multirow[b]{2}{*}{ Reference electrode } & \multirow[b]{2}{*}{ Site } & \multirow[b]{2}{*}{ Intensity (mA) } & \multirow[b]{2}{*}{ Size } & \multirow[b]{2}{*}{ Duration (min) } \\
\hline & iH & $\mathrm{CH}$ & Both & Sham & & & & & \\
\hline Bolognini et $a l^{18}$ & & & $\mathrm{~A} / \mathrm{C}$ & $\sqrt{ }$ & NA & M1 & 2 & $35 \mathrm{~cm}^{2}$ & 40 \\
\hline Celnik et $a l^{19}$ & $A$ & & & $\sqrt{ }$ & CSO & M1 & 1 & $57.8 \mathrm{~cm}^{2}$ & 20 \\
\hline Di Lazzaro et a ${ }^{25}$ & & & $\mathrm{~A} / \mathrm{C}$ & $\sqrt{ }$ & NA & M1 & 2 & $35 \mathrm{~cm}^{2}$ & 40 \\
\hline Fusco et $a l^{20}$ & & C & & $\sqrt{ }$ & CSD & M1 & 1.5 & $35 \mathrm{~cm}^{2}$ & 10 \\
\hline Geroin et $a l^{21}$ & $A$ & & & $\sqrt{ }$ & CSO & M1 (leg area) & 1.5 & $35 \mathrm{~cm}^{2}$ & 7 \\
\hline Hesse et $a l^{22}$ & $A$ & C & & $\sqrt{ }$ & CSO & M1 & 2 & $35 \mathrm{~cm}^{2}$ & 20 \\
\hline Khedr et $a l^{23}$ & $A$ & C & & $\sqrt{ }$ & CSO & M1 & 2 & $35 \mathrm{~cm}^{2}$ & 25 \\
\hline Kim et $a l^{24}$ & $A$ & $\mathrm{C}$ & & $\sqrt{ }$ & CSO & M1 & 2 & $25 \mathrm{~cm}^{2}$ & 20 \\
\hline Lefebvre et $\left.a\right|^{26}$ & & & $\mathrm{~A} / \mathrm{C}$ & $\sqrt{ }$ & NA & M1 & 1 & $35 \mathrm{~cm}^{2}$ & 30 \\
\hline Lefebvre et $a l^{27}$ & & & $\mathrm{~A} / \mathrm{C}$ & $\sqrt{ }$ & NA & M1 & 1 & $35 \mathrm{~cm}^{2}$ & 30 \\
\hline Lindenberg et $\left.a\right|^{28}$ & & & $\mathrm{~A} / \mathrm{C}$ & $\sqrt{ }$ & NA & M1 & 1.5 & $16.3 \mathrm{~cm}^{2}$ & 30 \\
\hline Mortensen et $a l^{33}$ & A & & & $\sqrt{ }$ & CSO & M1 & 1.5 & $35 \mathrm{~cm}^{2}$ & 20 \\
\hline Nair et $a l^{29}$ & & C & & $\sqrt{ }$ & CSO & M1 & 1 & NA & 30 \\
\hline Rocha et $a l^{34}$ & $A$ & $\mathrm{C}$ & & $\sqrt{ }$ & CSO & M1 & 1 & $35 \mathrm{~cm}^{2}$ & 13 (anodal), 9 (cathodal) \\
\hline Sattler et $a l^{32}$ & A & & & $\sqrt{ }$ & NA & M1 & 1.2 & $35 \mathrm{~cm}^{2}$ & 13 \\
\hline Wu et $a l^{30}$ & $\mathrm{C}$ & & & $\sqrt{ }$ & CSD & S1M1 & 1.2 & $24.8 \mathrm{~cm}^{2}$ & 20 \\
\hline Zimerman et $a l^{31}$ & & C & & $\sqrt{ }$ & CSO & M1 & 1 & $25 \mathrm{~cm}^{2}$ & 20 \\
\hline
\end{tabular}

A, anodal tDCS; C, cathodal tDCS; $C H$, contralesional hemisphere; CSD, contralateral shoulder; CSR, contralateral supraorbital region; iH, ipsilesional hemisphere; M1, primary motor cortex; NA, not available; S1M1, primary sensorimotor cortex; tDCS, transcranial direct current stimulation.

of the true effects sizes in a random effects model. ${ }^{39} \mathrm{~A} \mathrm{~T}^{2}$ greater than 1.0 denotes substantial heterogeneity between studies.

We examined publication bias with three statistical procedures that were consistent with traditional meta-analysis ${ }^{44}{ }^{45}$ : (1) funnel plot showing the symmetry of the studies (standardised mean differences vs SE for each comparison), ${ }^{42} 46$ (2) trim and fill technique for generating a subsequent funnel plot with imputed values to estimate an unbiased distribution ${ }^{47}$ and (3) classic fail-safe $\mathrm{N}$ analysis to determine the number of studies necessary to decrease the overall ES to an insignificant level. ${ }^{48}$

\section{RESULTS}

\section{Standardised mean difference effect}

A random effects model meta-analysis on the 21 comparisons showed a significant overall $\mathrm{ES}$ equal to $0.59 \quad(\mathrm{SE}=0.10$; $\mathrm{p}<0.0001 ; \mathrm{Z}=5.95 ; 95 \%$ CI 0.40 to 0.79 ). This is a positive medium ES. ${ }^{41}$ Table 4 displays each ES: minimum $=0.04$ and maximum $=1.59$. No individual weighted effect exceeded two SDs of the standardised mean ES. Figure 1 shows the individual comparisons in a forest plot. These robust findings indicate that tDCS improved motor learning post-stroke across stimulation protocols and stages of recovery. Moderator variable analyses provide further insights.

\section{Heterogeneity and publication bias}

Variability measures on the 21 comparisons revealed low heterogeneity: $\mathrm{Q}=25.89$ and $\mathrm{p}=0.17 ; \mathrm{I}^{2}=22.75 \% ; \mathrm{T}^{2}=0.04$ (table 4). Visual inspection of the funnel plot shows a relatively symmetrical distribution of each ES over the 21 comparisons (minor publication bias; figure 2). Applying the trim and fill method ${ }^{47}$ produced a relatively identical overall ES (figure 3: black diamond; no trimmed studies) with two imputed values in comparison to the original (white diamond). Moreover, a classic failsafe $\mathrm{N}$ analysis indicated that 240 null effect findings are required for decreasing our significant overall ES (0.59; $\mathrm{p}<0.0001)$ to an insignificant level $(\mathrm{p}>0.05)$. Consequently, these combined findings support a minor publication bias conclusion.

\section{Moderator variable analyses \\ Stimulation protocols}

The first moderator variable analysis investigated the effectiveness of three stimulation protocols on motor learning poststroke: (1) anodal stimulation on M1 of ipsilesional hemisphere, (2) cathodal stimulation on M1 of contralesional hemisphere and (3) bilateral (anodal+cathodal) stimulation on both hemispheres. Further, given that one comparison reported cathodal stimulation on S1M1 of ipsilesional hemisphere, we excluded this comparison from the subgroup analysis. Eight anodal stimulation comparisons revealed an $\mathrm{ES}=0.59(\mathrm{SE}=0.20 ; \mathrm{p}=0.003$; $\mathrm{Z}=2.98 ; 95 \%$ CI 0.20 to $0.97 ; \mathrm{I}^{2}=39.86 \% ; \mathrm{T}^{2}=0.12$ ) whereas seven cathodal stimulation comparisons showed an $\mathrm{ES}=0.60$ $(\mathrm{SE}=0.23 ; \mathrm{p}=0.009 ; \mathrm{Z}=2.62 ; 95 \% \quad \mathrm{CI} \quad 0.15$ to 1.04 ; $\left.\mathrm{I}^{2}=37.61 \% ; \mathrm{T}^{2}=0.13\right)$. Analysis of the five bilateral stimulation comparisons indicated an $\mathrm{ES}=0.68 \quad(\mathrm{SE}=0.16 ; \mathrm{p}<0.0001$; $\mathrm{Z}=4.29 ; 95 \%$ CI 0.37 to $\left.0.99 ; \mathrm{I}^{2}=0.00 \% ; \mathrm{T}^{2}=0.00\right)$. Taken together, these findings indicate beneficial effects of tDCS on motor learning post-stroke for each of the three stimulation protocols.

\section{Stage of recovery post-stroke}

In a second moderator analysis, we compared the long-term motor learning effects of tDCS based on three stages of poststroke recovery. However, only two acute comparisons were available in our meta-analysis. Rather than report a spurious finding for the acute phase, we did not analyse the earliest stage post-stroke. Analysis on the two subsequent stages showed significant standardised mean differences for (1) subacute stage (7 comparisons): $\mathrm{ES}=0.68$ ( $\mathrm{SE}=0.27 ; \mathrm{p}=0.01 ; \mathrm{Z}=2.53 ; 95 \%$ CI 0.15 to $\left.1.20 ; \mathrm{I}^{2}=64.45 \% ; \mathrm{T}^{2}=0.30\right)$ and $(2)$ chronic stage (12 comparisons): $\mathrm{ES}=0.64$ ( $\mathrm{SE}=0.11 ; \mathrm{p}<0.0001 ; \mathrm{Z}=6.02$; $95 \%$ CI 0.44 to $\left.0.85 ; \mathrm{I}^{2}=0.00 \% ; \mathrm{T}^{2}=0.00\right)$. This evidence 


\begin{tabular}{|c|c|c|c|c|c|c|c|}
\hline Study & Retention period & Outcome measure & $\mathrm{Rx} / \mathrm{Ctrl}(\mathrm{N})$ & $E S=S M D$ & $95 \% \mathrm{Cl}$ & & Relative weight \\
\hline Bolognini et $a l^{18}$ & 4 weeks & $\begin{array}{l}\text { Total latency score in JHFT (atDCS on } \mathrm{H}+\mathrm{CtDCS} \text { on } \mathrm{CH} \\
\text { during CIMT at retention: } \mathrm{Rx} \text { vs atDCS on } \mathrm{iH}+\mathrm{CtDCS} \text { on } \mathrm{cH} \\
\text { during CIMT at retention: } \mathrm{Ctrl} \text { ) }\end{array}$ & $7 / 7$ & 0.16 & -0.89 & 1.21 & 2.98 \\
\hline Celnik et $a l^{19}$ & 6 days & $\begin{array}{l}\text { Mean improvements of correct key press (PNS+atDCS on iH } \\
\text { before motor practise at retention: Rx vs PNS+sham before } \\
\text { motor practise at retention: Ctrl) }\end{array}$ & $9 / 9$ & 0.18 & -0.48 & 0.84 & 6.28 \\
\hline Di Lazzaro et a ${ }^{25}$ & 12 weeks & $\begin{array}{l}\text { NIHSS (atDCS on } \mathrm{iH}+\mathrm{ctDCS} \text { on } \mathrm{CH} \text { during } \mathrm{CIMT} \text { at } \\
\text { retention: } \mathrm{Rx} \text { vs sham during CIMT at retention: } \mathrm{Ctrl} \text { ) }\end{array}$ & $10 / 10$ & 0.36 & -0.52 & 1.24 & 3.99 \\
\hline Fusco et $a l^{20}$ & 4 weeks & $\begin{array}{l}\text { Filling speed in 9HPT (ctDCS on } \mathrm{cH} \text { before inpatient daily } \\
\text { rehabilitation at retention: } \mathrm{Rx} \text { vs sham before inpatient } \\
\text { daily rehabilitation at retention: } \mathrm{Ctrl} \text { ) }\end{array}$ & $5 / 6$ & 0.08 & -1.11 & 1.27 & 2.40 \\
\hline Geroin et $a^{21}$ & 2 weeks & $\begin{array}{l}\text { Six-minute walking test }(\mathrm{m}) \text { (atDCS on iH during } \\
\text { robot-assisted gait training at retention: Rx vs sham during } \\
\text { robot-assisted gait training at retention: Ctrl) }\end{array}$ & $10 / 10$ & 0.38 & -0.50 & 1.27 & 3.98 \\
\hline \multirow[t]{2}{*}{ Hesse et $a l^{22}$} & 12 weeks & $\begin{array}{l}\text { FMA (atDCS on iH during bilateral arm robot training at } \\
\text { retention: Rx vs sham during bilateral arm robot training at } \\
\text { retention: Ctrl) }\end{array}$ & $28 / 28$ & 0.04 & -0.48 & 0.56 & 8.53 \\
\hline & & $\begin{array}{l}\text { FMA (ctDCS on } \mathrm{cH} \text { during bilateral arm robot training at } \\
\text { retention: } \mathrm{Rx} \text { vs sham during bilateral arm robot training at } \\
\text { retention: } \mathrm{Ctrl} \text { ) }\end{array}$ & $29 / 28$ & 0.06 & -0.46 & 0.58 & 8.62 \\
\hline \multirow[t]{2}{*}{ Khedr et $a l^{23}$} & 12 weeks & $\begin{array}{l}\text { NIHSS (atDCS on iH before motor training at retention: Rx } \\
\text { vs sham before motor training at retention: Ctrl) }\end{array}$ & $14 / 13$ & 1.59 & 0.73 & 2.46 & 4.12 \\
\hline & & $\begin{array}{l}\text { NIHSS (ctDCS on } \mathrm{cH} \text { before motor training at retention: } \mathrm{Rx} \\
\text { vs sham before motor training at retention: }(\mathrm{trl})\end{array}$ & $13 / 13$ & 1.08 & 0.26 & 1.90 & 4.47 \\
\hline \multirow[t]{2}{*}{ Kim et $a l^{24}$} & 24 weeks & $\begin{array}{l}\text { FMA (atDCS on iH during OT at retention: Rx vs sham } \\
\text { during OT at retention: Ctrl) }\end{array}$ & $6 / 7$ & 1.05 & -0.11 & 2.21 & 2.49 \\
\hline & & $\begin{array}{l}\text { FMA (ctDCS on } \mathrm{CH} \text { during } \mathrm{OT} \text { at retention: } \mathrm{Rx} \text { vs sham } \\
\text { during OT at retention: } \mathrm{Ctrl} \text { ) }\end{array}$ & $5 / 7$ & 1.39 & 0.11 & 2.66 & 2.11 \\
\hline Lefebvre et $a^{26}$ & 1 week & $\begin{array}{l}\text { Learning index in motor skill learning task (atDCS on iH } \\
+\mathrm{CtDCS} \text { on } \mathrm{cH} \text { during motor skill learning task at retention: } \\
\text { Rx vs baseline: Ctrl) }\end{array}$ & $18 / 18$ & 0.93 & 0.38 & 1.49 & 7.95 \\
\hline Lefebvre et $a^{27}$ & 1 week & $\begin{array}{l}\text { Learning index in motor skill learning task (atDCS on iH } \\
+ \text { ctDCS on } \mathrm{CH} \text { during motor skill learning task at retention: } \\
\text { Rx vs baseline: Ctrl) }\end{array}$ & $19 / 19$ & 0.82 & 0.30 & 1.34 & 8.62 \\
\hline Lindenberg et al ${ }^{28}$ & 1 week & $\begin{array}{l}\text { FMA (atDCS on } \mathrm{iH}+\mathrm{CtDCS} \text { on } \mathrm{CH} \text { during } \mathrm{PT}+\mathrm{OT} \text { at } \\
\text { retention: } \mathrm{Rx} \text { vs sham during } \mathrm{PT}+\mathrm{OT} \text { at retention: } \mathrm{Ctrl} \text { ) }\end{array}$ & $10 / 10$ & 0.29 & -0.59 & 1.17 & 4.00 \\
\hline Mortensen et $a^{33}$ & 1 week & $\begin{array}{l}\text { Score in JHFT (atDCS on iH during OT at retention: Rx vs } \\
\text { sham during OT at retention: Ctrl) }\end{array}$ & $8 / 7$ & 0.61 & -0.43 & 1.65 & 3.04 \\
\hline Nair et a $\left.\right|^{29}$ & 1 week & $\begin{array}{l}\text { 3J-ROM (ctDCS on } \mathrm{CH} \text { during } \mathrm{OT} \text { at retention: } \mathrm{Rx} \text { vs sham } \\
\text { during OT at retention: } \mathrm{Ctrl} \text { ) }\end{array}$ & $7 / 7$ & 1.43 & 0.25 & 2.60 & 2.45 \\
\hline \multirow[t]{2}{*}{ Rocha et al ${ }^{34}$} & 4 weeks & $\begin{array}{l}\text { FMA (atDCS on iH before mCIMT at retention: Rx vs sham } \\
\text { before mCIMT at retention: Ctrl) }\end{array}$ & $7 / 7$ & 0.94 & -0.17 & 2.04 & 2.73 \\
\hline & & $\begin{array}{l}\text { FMA (ctDCS on } \mathrm{cH} \text { before } \mathrm{mCIMT} \text { at retention: Rx vs sham } \\
\text { before mCIMT at retention: } \mathrm{Ctrl} \text { ) }\end{array}$ & $7 / 7$ & 0.24 & -0.81 & 1.29 & 2.97 \\
\hline Sattler et $a^{\beta 2}$ & 4 weeks & $\begin{array}{l}\text { Time in JHFT (rPNS+atDCS on iH before OT at retention: Rx } \\
\text { vs rPNS+sham before OT at retention: Ctrl) }\end{array}$ & $10 / 10$ & 0.65 & -0.25 & 1.55 & 3.87 \\
\hline
\end{tabular}




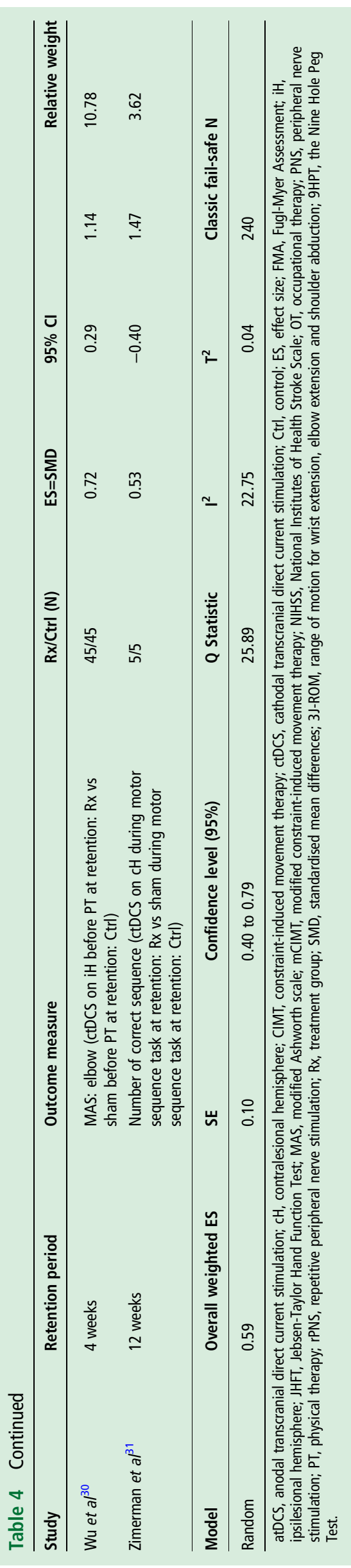

points to long-term motor learning effects of tDCS in patients with subacute and chronic stroke.

\section{Stimulation timing}

A third moderator analysis examined the effects of stimulation timing on motor learning. Direct comparison of stimulation before versus during motor training involved all 21 comparisons. Analysis of the eight comparisons that provided stimulation before motor training revealed an $\mathrm{ES}=0.70 \quad(\mathrm{SE}=0.17$; $\mathrm{p}<0.0001 ; \mathrm{Z}=4.21 ; 95 \%$ CI 0.37 to $1.02 ; \mathrm{I}^{2}=24.30 \%$; $\mathrm{T}^{2}=0.05$ ). On the other hand, the 13 comparisons used a tDCS protocol during motor training found a standardised mean difference $=0.53(\mathrm{SE}=0.13 ; \mathrm{p}<0.0001 ; \mathrm{Z}=4.19 ; 95 \%$ CI 0.28 to $\left.0.77 ; \mathrm{I}^{2}=22.27 \% ; \mathrm{T}^{2}=0.04\right)$. The results clearly indicate longterm motor learning for stimulation protocols either before or during motor training practise.

Further, tDCS after effects may have contributed to motor improvements once the stimulation duration ended, especially with extended motor practise. Thus, we performed an additional moderator variable analysis on the motor practise durations in the stimulation during condition. This analysis evaluated the eight comparisons that continued motor practise beyond the simulation interval versus the five that stopped motor practise when the stimulation ended. We found two significant ES: (1) continued motor practise beyond tDCS: $\mathrm{ES}=0.61(\mathrm{SE}=0.18 ; \mathrm{p}=0.001 ; \mathrm{Z}=3.31 ; 95 \%$ CI 0.25 to 0.97 ; $\left.\mathrm{I}^{2}=0.00 \% ; \mathrm{T}^{2}=0.00\right)$ and (2) stopped motor practise at the end of stimulation: $\mathrm{ES}=0.47(\mathrm{SE}=0.20 ; \mathrm{p}=0.02 ; \mathrm{Z}=2.29 ; 95 \% \mathrm{CI}$ 0.07 to $0.87 ; \mathrm{I}^{2}=57.42 \% ; \mathrm{T}^{2}=0.12$ ). These two moderator variable findings support a conservative conclusion that a potential confound from stimulation after effects minimally influenced the motor learning treatment effect found in the tDCS during motor training condition.

\section{Task-specific training versus conventional rehabilitation protocols}

The final moderator analysis focused on the effects of tDCS on long-term motor learning combined with task-specific training versus conventional rehabilitation. The analysis revealed significant ES for both types of training: (1) tDCS with task-specific training: $\mathrm{ES}=0.65 \quad(\mathrm{SE}=0.15 ; \mathrm{p}<0.0001 ; \mathrm{Z}=4.36 ; 95 \% \mathrm{CI}$ 0.36 to $0.94 ; \mathrm{I}^{2}=0.00 \% ; \mathrm{T}^{2}=0.00 ; 5$ comparisons) and (2) tDCS with conventional rehabilitation protocols: $\mathrm{ES}=0.59$ $(\mathrm{SE}=0.13 ; \mathrm{p}<0.0001 ; \mathrm{Z}=4.55 ; 95 \% \quad \mathrm{CI} \quad 0.34$ to 0.84 ; $\mathrm{I}^{2}=31.16 \% ; \mathrm{T}^{2}=0.08 ; 16$ comparisons). Both moderator variable findings support tDCS combined with either task-specific training or conventional rehabilitation for long-term motor learning post-stroke.

\section{DISCUSSION}

This focused systematic review and meta-analysis investigated long-term motor learning in stroke individuals after tDCS interventions combined with motor training. Making progress towards restoring voluntary movements by measuring motor learning improvements from baseline to long-term retention testing is crucial for understanding functional recovery of stroke survivors. Together, the meta-analytic techniques conducted on the 21 comparisons from 17 qualified studies support the conclusion that the tDCS protocols showed long-term beneficial effects on voluntary/goal-directed movements post-stroke. These significant, positive and robust tDCS findings revealed substantial motor learning improvements for individuals in the subacute and chronic stages of recovery. Moreover, analysis of tDCS protocols either provided before or during motor training revealed similar beneficial long-term effects on motor functions post-stroke. 
Bolognini et al ${ }^{18}$ Celnik et $a l^{19}$

Di Lazzaro et a ${ }^{25}$

Fusco et a ${ }^{20}$

Geroin et a ${ }^{21}$

Hesse et al 22 (anodal)

Hesse et a ${ }^{22}$ (cathodal)

Khedr et al ${ }^{23}$ (anodal)

Khedr et a ${ }^{23}$ (cathodal)

Kim et $a^{24}$ (anodal)

Kim et a ${ }^{24}$ (cathodal)

Lefebvre et al ${ }^{26}$

Lefebvre $e a^{27}$

Lindenberg et $a^{28}$

Mortensen $e^{a} \mathrm{l}^{3}$

Nair et a ${ }^{29}$

Rocha et $^{\beta{ }^{34}}$ (anodal)

Rocha et $a^{\beta 4}$ (cathodal)

Sattler et $a^{\beta 2}$

Wu et $a^{30}$

Zimerman $e^{a}{ }^{\beta 1}$

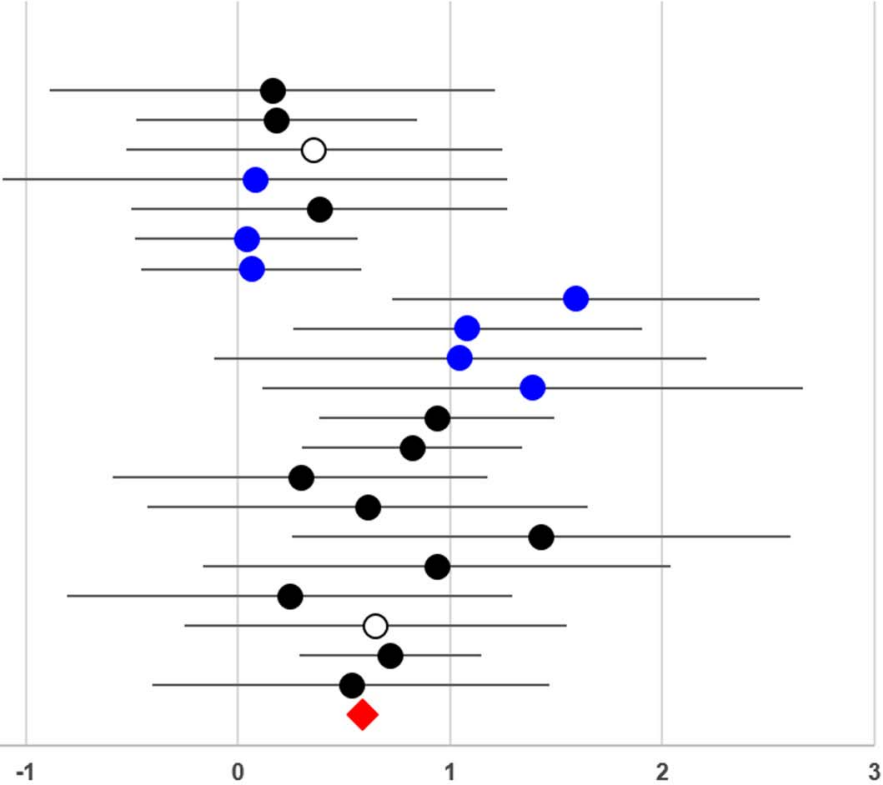

Figure 1 Meta-analysis forest plot of the effects of transcranial direct current stimulation on motor learning post-stroke. Data derived from a random effects model. Each line and tick mark represents an individual effect size. The red diamond indicates an overall effect size equal to 0.59 . Circle colours: black=chronic; blue=subacute; white=acute.

\section{Stimulation protocols}

Three significant ES from each stimulation protocol indicated that the three tDCS protocols contribute to long-term motor learning improvements post-stroke. Brasil-Neto ${ }^{49}$ reported a possibility that tDCS interventions facilitated long-term motor improvements in healthy individuals and patients because of the contribution of tDCS (anodal and cathodal stimulation) to longterm potentiation and long-term depression. Balancing cortical activities between hemispheres by (1) anodal stimulation on M1 of ipsilesional hemisphere, (2) cathodal stimulation on M1 of contralesional hemisphere and (3) bilateral stimulation on both hemispheres is likely to improve long-term motor improvements. ${ }^{50}$ However, Bestmann et $a l^{51}$ argued that brain regions (ie, premotor cortex and supplementary motor cortex) interacting with the tDCS target area (ie, M1) potentially contribute to behavioural improvements. Further, Sehm et al $l^{52}$ reported that increased interconnectivity between M1 and other brain areas was associated with improved motor functions. Specifically, Lefebvre $e t a l^{27}$ revealed that adaptive recruitment of the premotor cortex in the ipsilesional hemisphere (ie, less activation patterns after bilateral stimulation than the sham control group) may be associated with motor learning improvements in stroke
Figure 2 Funnel plot of the comparisons for random effects model. The $x$-axis represents the standardised mean difference and the $y$-axis indicates the SE associated with each comparison.

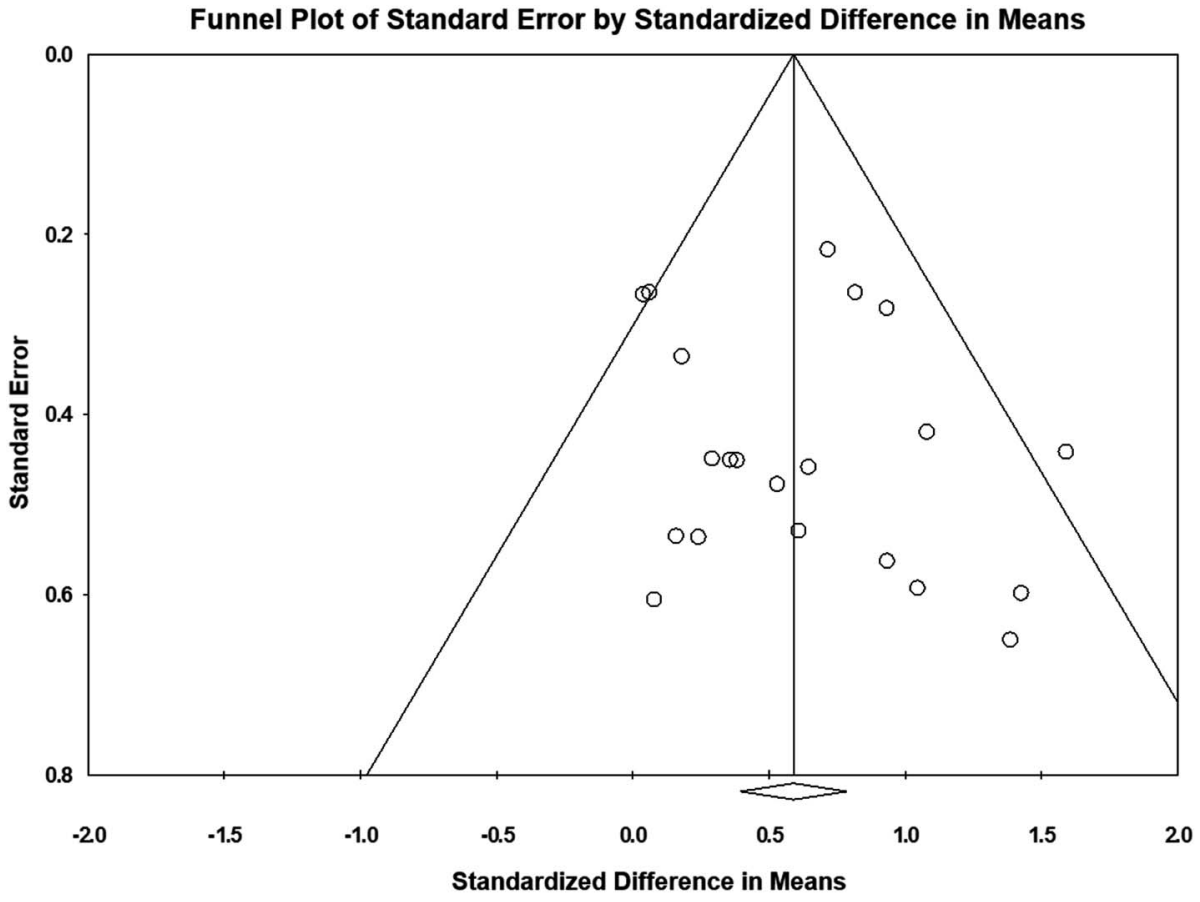


Figure 3 Best estimate funnel plot of a symmetrical funnel unbiased effect. White circles and white diamond indicate our original 21 comparisons while the black circles and black diamond represent imputed comparisons after the trim and fill technique.

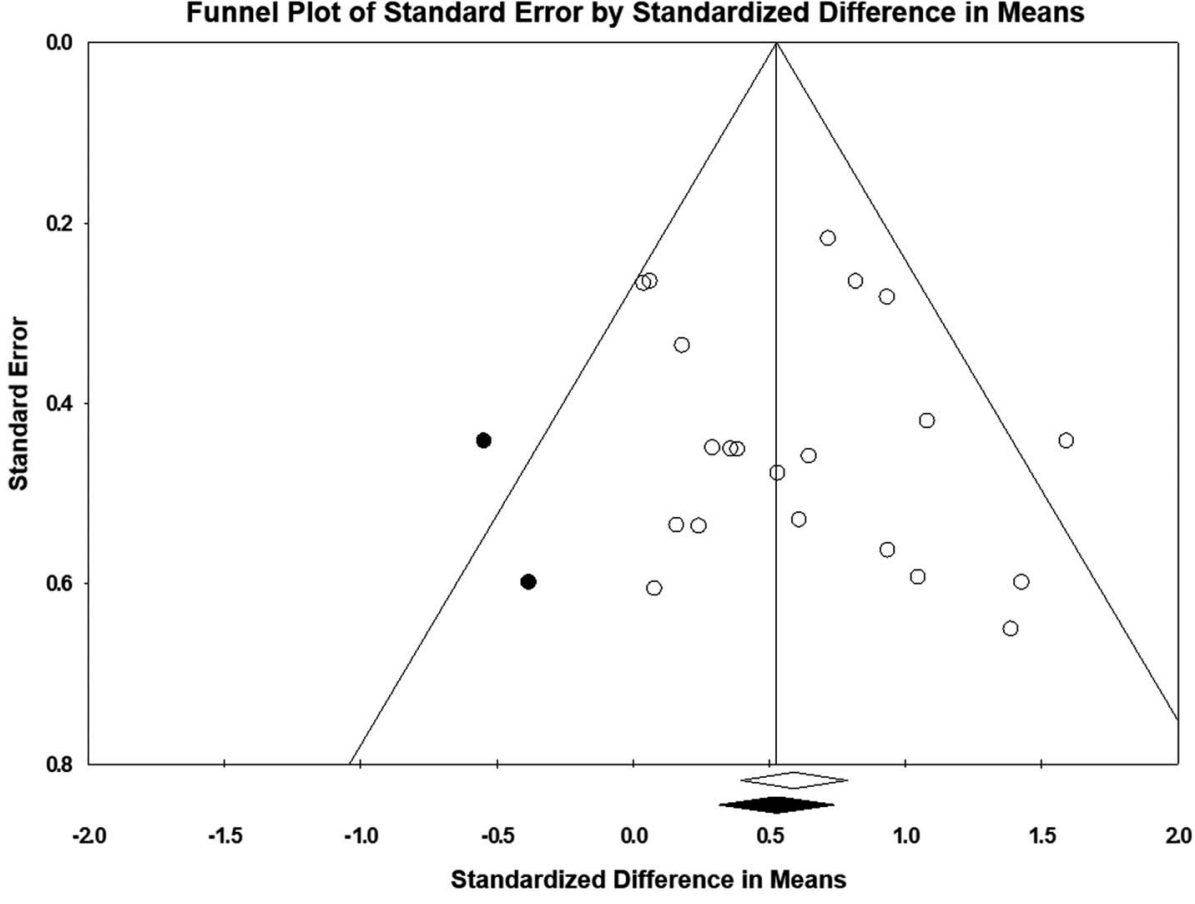

survivors. A conservative conclusion is that the potential mechanisms underlying long-term effects of tDCS protocols on stroke motor functions involve modulation of brain activity in M1 as well as other brain areas of both hemispheres. ${ }^{53}$

\section{Post-stroke recovery stages}

Beneficial long-term motor learning effects appeared in the subacute and chronic recovery stages post-stroke. Although our meta-analytic techniques found a significant positive ES in the subacute group (seven comparisons; $E S=0.68$ ), caution should be exercised because of the high heterogeneity $\left(\mathrm{I}^{2}=64.45 \%\right)$ and wider confidence level (95\% CI 0.15 to 1.20$).{ }^{39}$ In fact, three comparisons in Fusco et $a l^{20}$ and Hesse et $a l^{22}$ reported non-significant individual ES around zero whereas four comparisons in Khedr et $a l^{23}$ and Kim et $a l^{24}$ showed higher significant individual ES (>1.0). On the other hand, long-term effects of tDCS protocols on chronic stroke are robust $(\mathrm{ES}=0.64 ; 95 \% \mathrm{CI}$ 0.44 to $\left.0.85 ; \mathrm{I}^{2}=0.00 \%\right)$. Positive effects of tDCS on chronic patients are consistent with two previous systematic reviews and meta-analyses in that significant motor improvements post-tDCS interventions are most evident in the chronic stage of recovery. ${ }^{79}$ Based on the interhemispheric competition model, 16 qualified studies (except for Wu et $a l^{30}$ ) used anodal stimulation on the ipsilesional hemisphere, cathodal stimulation on the contralesional hemisphere or bilateral stimulation across a range of stroke participants who may have differed in terms of impairment severity, lesion location (eg, cortical and subcortical) or recovery stage (eg, acute, subacute and chronic). However, the three tDCS protocols (ie, anodal, cathodal and bilateral stimulation) applied to different levels of stroke severity may cause interindividual variability in the efficacy of rehabilitation programmes. $^{5455}$

\section{Potential mechanisms}

A frequent assertion about the mechanism underlying stroke motor recovery post-tDCS centres on the controversial interhemispheric competition model. ${ }^{5657}$ After a stroke, the magnitude of interhemispheric inhibition from the contralesional hemisphere is usually greater than the excitability of the ipsilesional hemisphere. Inhibition from the contralesional hemisphere interferes with the level of cortical excitability in the ipsilesional hemisphere causing paretic limb impairment. This model maintains that balancing both excitatory and inhibitory activation between hemispheres via tDCS protocols (ie, anodal stimulation on ipsilesional hemisphere, cathodal stimulation on contralesional hemisphere, bilateral stimulation on both hemispheres) facilitates functional recovery in stroke survivors. That is, suppressing brain activity in the contralesional hemisphere facilitates paretic limb recovery.

In contrast, an alternative model called vicariation asserts that cortical excitability in the contralesional hemisphere compensates for impaired functions of the ipsilesional hemisphere while performing paretic hand movements. ${ }^{58} 59$ The increased brain activity in the contralesional hemisphere during paretic arm movements contributes to motor recovery. ${ }^{60}$ Hummel et al ${ }^{60}$ stated that cathodal stimulation on the contralesional hemisphere is disadvantageous for some patients with stroke because brain activity patterns in the contralesional hemisphere were activated while executing paretic hand movements for some patients. $^{6162}$ Indeed, Wu et al's ${ }^{30}$ novel technique identified an unusual finding; cathodal stimulation on the S1M1 of the ipsilesional hemisphere improved paretic arm function by suppressing the hyperactivation typically seen in this area.

An attempt to resolve the controversy surrounding the mechanisms involved in tDCS and post-stroke motor learning was proposed by Di Pino et al. ${ }^{55}$ Their integrated rehabilitation model referred to as bimodal balance-recovery model includes components of both vicariation and interhemispheric competition models. Moreover, the bimodal balance-recovery model introduces structure reserve, the quantity of strategic neural pathways and relays spared by the lesion. Higher structure reserve typically indicates better motor recovery than lower structure reserve. ${ }^{55}$ The bimodal balance-recovery model posits that the vicariation model accurately predicts recovery for patients with lower structure reserve (eg, large affected area and severe damage) whereas the interhemispheric competition 
model is more appropriate for patients with higher structure reserve (eg, smaller affected area and minimal damage). Thus, the behavioural benefits from the three types of tDCS protocols based on the interhemispheric competition model may decrease for patients with lower structure reserve. In line with previous findings, the motor learning ES found for our chronic group indicates that recovery stage may influence the structure reserve causing interindividual variability in the efficacy of uniform tDCS protocols. Applying individualised tDCS protocols based on stroke severity and stroke region extent may minimise interindividual variability in tDCS treatment effects.

A third viable argument considers individualised current modelling. Bestmann et $a l^{5163}$ posit that computational neurostimulation is necessary to normalise current flow for participants who have different anatomical structures. tDCS computational modelling of current flows provides specific details concerning induced physiological and behavioural changes. Moreover, Brunoni et $a l^{53}$ suggested two approaches for increasing the accuracy of the computational modelling for current flow: (1) using high-resolution anatomic scans and (2) applying a priori knowledge about tissue anatomy. Taken together, an important consequence of this computational modelling is determining tDCS protocol efficacy. ${ }^{53}$

\section{Additional issues: stimulation timing and task specificity in motor training}

A third moderator variable analysis showed that both tDCS before and during motor training significantly facilitate longterm motor improvements post-stroke. The ES found for stimulation before motor training $(E S=0.70)$ was slightly higher than stimulation during motor training $(E S=0.53)$. However, the optimal timing of tDCS (ie, before vs during motor training) is still open to question. Giacobbe $e t a l^{64}$ reported that movement smoothness improved when tDCS was applied before movement training, whereas no improvement was found in tDCS during movement training. On the other hand, Stagg et al ${ }^{65}$ reported slower motor learning when both anodal and cathodal stimulations were provided before the motor learning task. Further, given that different brain activation mechanisms based on stimulation timing (ie, before vs during motor practise), ${ }^{66}{ }^{67}$ additional studies investigating the order of tDCS protocols while practising voluntary movements are necessary to further elaborate on maximising motor learning effects post-stroke.

In addition, given that $\mathrm{tDCS}$ protocols during motor practise typically continued the motor training beyond the tDCS duration, after effects of tDCS may have contributed to motor improvements once the tDCS duration ended. In our current meta-analytic findings, 8 of the 13 comparisons (ie, tDCS during motor training) continued motor practise after the end of the stimulation, whereas five comparisons matched the stimulation duration and motor practise interval. The mean duration of the continued motor practise beyond stimulation equalled $29.5 \mathrm{~min}(\mathrm{SD}=54.1 \mathrm{~min})$. This duration is relatively less than the mean duration of motor practise in the tDCS before motor training condition (mean=114.1 $\mathrm{min}$ and $\mathrm{SD}=151.8 \mathrm{~min}$ ). Given that the mean duration of motor practise for the before tDCS condition is nearly four times as long as the motor practise duration in the stimulation during motor training condition, arguing that the tDCS after effects from both motor practise conditions are similar is tenuous. Moreover, two significant medium ES between the two stimulation timing conditions (ie, continued motor practise beyond tDCS: $\mathrm{ES}=0.61$; stopped motor practise at the end of stimulation: $E S=0.47$ ) support our conclusion that a potential confound from stimulation after effects minimally influenced the motor learning treatment effect found in the tDCS during the motor practise condition.

Another moderator variable analysis investigated the longterm motor learning effects of tDCS with task-specific training and conventional rehabilitation. The positive ES for task-specific training $(E S=0.65)$ was slightly higher than tDCS with conventional rehabilitation $(\mathrm{ES}=0.59)$. These findings are consistent with Winstein $e$ e al's ${ }^{17}$ proposition that task specificity (ie, consistency between trained movements and desired goal-directed actions) during stroke motor rehabilitation is critical to increasing the treatment effects. However, tDCS protocols combined with conventional therapies revealed a significant moderate long-term motor learning effect in stroke survivors as well. These cumulative findings indicate that our motor learning evidence following tDCS interventions was robust regardless of the type of motor practise executed during training.

Despite the substantial motor learning effects of tDCS protocols on arm movements post-stroke, the number of studies focusing on lower extremity functions as well as the acute recovery stage is limited. Indeed, long-term motor learning effects of tDCS are still debateable for the lower extremities as well as the acute recovery stage. ${ }^{70}$ Conducting more long-term follow-up testing for both conditions may consolidate rehabilitative effects of tDCS interventions.

In conclusion, this systematic review and meta-analysis provides convincing evidence supporting a conclusion that tDCS and motor practise positively facilitated long-term motor learning in stroke individuals. Moreover, moderator variable analyses showed that long-term motor learning effects appeared following each of the three tDCS protocols (ie, anodal stimulation on M1 of the ipsilesional hemisphere, cathodal stimulation on M1 of the contralesional hemisphere and bilateral stimulation on both hemispheres). Additional tDCS studies investigating motor learning effects based on different structure reserve representations will be necessary for researchers to develop individualised tDCS protocols. Further, given that brain imaging studies confirm that transient motor improvements correlate with brain activation patterns modulated by tDCS, ${ }^{68-70}$ there is a need to investigate brain activation changes during tDCS-induced motor learning to identify the neurological mechanisms underlying long-term functional recovery post-stroke.

Acknowledgements JJS gratefully acknowledges funding from the Australian Research Council Discovery Projects scheme (DP130104317). Funding from the American Heart Association (USA) is gratefully acknowledged in supporting this research with a grant to JHC (\#00093013).

Contributors NK independently extracted relevant data from the 17 studies and coded the outcome measures. JJS and JHC confirmed the extracted data. Each author contributed to interpreting the meta-analytic results, manuscript drafts, and approved revisions and the final manuscript.

Funding Australian Research Council Discovery Projects scheme (DP130104317) and American Heart Association (00093013).

Competing interests None declared.

Provenance and peer review Not commissioned; externally peer reviewed.

\section{REFERENCES}

1 Mozaffarian D, Benjamin EJ, Go AS, et al. Heart disease and stroke statistics2015 update: a report from the American Heart Association. Circulation 2015;131: e29-322.

2 Sainburg R, Good D, Przybyla A. Bilateral synergy: a framework for post-stroke rehabilitation. I Neurol Transl Neurosci 2013;1:1025.

3 Bindman LJ, Lippold OC, Redfearn JW. The action of brief polarizing currents on the cerebral cortex of the rat (1) during current flow and (2) in the production of long-lasting after-effects. J Physiol 1964;172:369-82. 
4 Gartside IB. Mechanisms of sustained increases of firing rate of neurons in the rat cerebral cortex after polarization: reverberating circuits or modification of synaptic conductance? Nature 1968;220:382-3.

5 Nitsche MA, Paulus W. Excitability changes induced in the human motor cortex by weak transcranial direct current stimulation. J Physiol 2000;527:633-9.

6 Nitsche MA, Paulus W. Sustained excitability elevations induced by transcranial DC motor cortex stimulation in humans. Neurology 2001;57:1899-901.

7 Ludemann-Podubecka J, Bosl K, Rothhardt S, et al. Transcranial direct current stimulation for motor recovery of upper limb function after stroke. Neurosc Biobehav Rev 2014;47:245-59.

8 Adeyemo BO, Simis M, Macea DD, et al. Systematic review of parameters of stimulation, clinical trial design characteristics, and motor outcomes in non-invasive brain stimulation in stroke. Front Psychiatry 2012;3:88.

9 Marquez J, Van Vliet $\mathrm{P}, \mathrm{McElduff} \mathrm{P}$, et al. Transcranial direct current stimulation (tDCS): does it have merit in stroke rehabilitation? A systematic review. Int I Stroke 2015; 10:306-16.

10 Elsner B, Kugler J, Pohl M, et al. Transcranial direct current stimulation (tDCS) for improving function and activities of daily living in patients after stroke. Cochrane Database Syst Rev 2013:CD009645.

11 Anderson JR. Learning and memory. New York: Wiley, 2000.

12 Schmidt RA, Lee TD. Motor learning and performance. 5th edn. Champaign: Human Kinetics, 2013.

13 Winstein CJ. Knowledge of results and motor learning-implications for physical therapy. Phys Ther 1991;71:140-9.

14 Krakauer JW. Motor learning: its relevance to stroke recovery and neurorehabilitation. Curr Opin Neurol 2006;19:84-90.

15 Bastian AJ. Understanding sensorimotor adaptation and learning for rehabilitation. Curr Opin Neurol 2008;21:628-33.

16 Choi JT, Bastian AJ. Adaptation reveals independent control networks for human walking. Nat Neurosci 2007;10:1055-62.

17 Winstein CJ, Rose DK, Tan SM, et al. A randomized controlled comparison of upper-extremity rehabilitation strategies in acute stroke: a pilot study of immediate and long-term outcomes. Arch Phys Med Rehabil 2004;85:620-8.

18 Bolognini N, Vallar G, Casati C, et al. Neurophysiological and behavioral effects of tDCS combined with constraint-induced movement therapy in poststroke patients. Neurorehabil Neural Repair 2011;25:819-29.

19 Celnik P, Paik NJ, Vandermeeren $Y$, et al. Effects of combined peripheral nerve stimulation and brain polarization on performance of a motor sequence task after chronic stroke. Stroke 2009;40:1764-71.

20 Fusco A, Assenza F, losa M, et al. The ineffective role of cathodal tDCS in enhancing the functional motor outcomes in early phase of stroke rehabilitation: an experimental trial. Biomed Res Int 2014;2014:547290.

21 Geroin C, Picelli A, Munari D, et al. Combined transcranial direct current stimulation and robot-assisted gait training in patients with chronic stroke: a preliminary comparison. Clin Rehabil 2011;25:537-48.

22 Hesse S, Waldner A, Mehrholz J, et al. Combined transcranial direct current stimulation and robot-assisted arm training in subacute stroke patients: an exploratory, randomized multicenter trial. Neurorehabil Neural Repair 2011;25:838-46.

23 Khedr EM, Shawky OA, El-Hammady DH, et al. Effect of anodal versus cathodal transcranial direct current stimulation on stroke rehabilitation: a pilot randomized controlled trial. Neurorehabil Neural Repair 2013;27:592-601.

24 Kim DY, Lim JY, Kang EK, et al. Effect of transcranial direct current stimulation on motor recovery in patients with subacute stroke. Am J Phys Med Rehabil 2010;89:879-86.

25 Di Lazzaro VD, Dileone M, Capone F, et al. Immediate and late modulation of interhemipheric imbalance with bilateral transcranial direct current stimulation in acute stroke. Brain Stimul 2014;7:841-8.

26 Lefebvre S, Laloux P, Peeters A, et al. Dual-tDCS enhances online motor skill learning and long-term retention in chronic stroke patients. Front Hum Neurosci 2013:6:343.

27 Lefebvre $S$, Dricot L, Laloux $P$, et al. Neural substrates underlying stimulation-enhanced motor skill learning after stroke. Brain 2015;138:149-63.

28 Lindenberg R, Renga V, Zhu LL, et al. Bihemispheric brain stimulation facilitates motor recovery in chronic stroke patients. Neurology 2010;75:2176-84.

29 Nair DG, Renga V, Lindenberg $R$, et al. Optimizing recovery potential through simultaneous occupational therapy and non-invasive brain-stimulation using tDCS Restor Neurol Neurosci 2011;29:411-20.

30 Wu DY, Qian L, Zorowitz RD, et al. Effects on decreasing upper-limb poststroke muscle tone using transcranial direct current stimulation: a randomized sham-controlled study. Arch Phys Med Rehabil 2013;94:1-8.

31 Zimerman $\mathrm{M}$, Heise KF, Hoppe J, et al. Modulation of training by single-session transcranial direct current stimulation to the intact motor cortex enhances motor skill acquisition of the paretic hand. Stroke 2012;43:2185-9.

32 Sattler V, Acket B, Raposo N, et al. Anodal tDCS combined with radial nerve stimulation promotes hand motor recovery in the acute phase after ischemic stroke. Neurorehabil Neural Repair Published Online First: 1 Jan 2015. doi:10.1177/ 1545968314565465
33 Mortensen J, Figlewski K, Andersen H. Combined transcranial direct current stimulation and home-based occupational therapy for upper limb motor impairment following intracerebral hemorrhage: a double-blind randomized controlled trial. Disabil Rehabil Published Online First: 16 Jun 2015. doi:10.3109/ 09638288.2015.1055379

34 Rocha S, Silva E, Foerster A, et al. The impact of transcranial direct current stimulation (tDCS) combined with modified constraint-induced movement therapy (mCIMT) on upper limb function in chronic stroke: a double-blind randomized controlled trial. Disabil Rehabil Published Online First: 10 Jun 2015. doi:10.3109/ 09638288.2015 .1055382

35 Ang KK, Guan C, Phua KS, et al. Facilitating effects of transcranial direct current stimulation on motor imagery brain-computer interface with robotic feedback for stroke rehabilitation. Arch Phys Med Rehabil 2015;96:S79-87.

36 Gresham GE, Duncan PW, Stason WB, et al. Post-stroke rehabilitation. Clinical practice guideline, No. 16. Rockville: US Department of Health and Human Services, Public Health Service, Agency for Health Care Policy and Research, 1995.

37 Fanelli D. How many scientists fabricate and falsify research? A systematic review and meta-analysis of survey data. PLOS ONE 2009;4:e5738.

38 Rosenthal R. Writing meta-analytic reviews. Psychol Bull 1995;118:183-92.

39 Borenstein M, Hedges LV, Higgins JPT, et al. Introduction to meta-analysis. New York: Wiley, 2009.

40 Borenstein M, Higgins JP. Meta-analysis and subgroups. Prev Sci 2013;14:134-43.

41 Rosenthal R, DiMatteo MR. Meta-analysis: recent developments in quantitative methods for literature reviews. Annu Rev Psychol 2001;52:59-82.

42 Higgins JPT, Green S. Cochrane handbook for systematic reviews of interventions 5.1.0. The Cochrane Collaboration, 2011. http://www.cochrane-handbook.org.

43 Higgins JPT, Thompson SG, Deeks JJ, et al. Measuring inconsistency in meta-analyses. BMJ 2003;327:557-60.

44 Hedges LV, Olkin I. Statistical methods for meta-analysis. Orlando: Academic Press, 1985.

45 Sutton AJ, Abrams KR, Jones DR, et al. Methods for meta-analysis in medical research. New York: Wiley, 2000.

46 Sterne JA, Egger M. Funnel plots for detecting bias in meta-analysis: guidelines on choice of axis. J Clin Epidemiol 2001;54:1046-55.

47 Duval S, Tweedie R. Trim and fill: a simple funnel-plot-based method of testing and adjusting for publication bias in meta-analysis. Biometrics 2000;56: 455-63.

48 Rosenthal R. The file drawer problem and tolerance for null results. Psychol Bull 1979;86:638-41.

49 Brasil-Neto JP. Learning, memory, and transcranial direct current stimulation. Front Psychiatry 2012;3:80.

50 Madhavan S, Shah B. Enhancing motor skill learning with transcranial direct current stimulation-a concise review with applications to stroke. Front Psychiatry 2012:3:66.

51 Bestmann S, de Berker AO, Bonaiuto J. Understanding the behavioural consequences of noninvasive brain stimulation. Trends Cogn Sci 2015;19:13-20.

52 Sehm B, Kipping J, Schafer A, et al. A comparison between uni- and bilateral tDCS effects on functional connectivity of the human motor cortex. Front Hum Neurosci 2013;7:183.

53 Brunoni AR, Nitsche MA, Bolognini N, et al. Clinical research with transcranial direct current stimulation (tDCS): challenges and future directions. Brain Stimul 2012;5:175-95

54 Bradnam LV, Stinear CM, Barber PA, et al. Contralesional hemisphere control of the proximal paretic upper limb following stroke. Cereb Cortex 2012;22:2662-71.

55 Di Pino G, Pellegrino G, Assenza G, et al. Modulation of brain plasticity in stroke: a novel model for neurorehabilitation. Nat Rev Neurol 2014;10:597-608.

56 Nowak DA, Grefkes C, Ameli M, et al. Interhemispheric competition after stroke: brain stimulation to enhance recovery of function of the affected hand. Neurorehabil Neural Repair 2009;23:641-56.

57 Murase N, Duque J, Mazzocchio R, et al. Influence of interhemispheric interactions on motor function in chronic stroke. Ann Neurol 2004;55:400-9.

58 Finger S. Chapter 51: recovery of function: redundancy and vicariation theories. Handb Clin Neurol 2010;95:833-41.

59 Jaillard A, Martin CD, Garambois K, et al. Vicarious function within the human primary motor cortex? Brain 2005;128:1122-38.

60 Hummel FC, Ceinik P, Pascual-Leone A, et al. Controversy: noninvasive and invasive cortical stimulation show efficacy in treating stroke patients. Brain Stimul 2008; 1:370-82

61 Gerloff C, Bushara K, Sailer A, et al. Multimodal imaging of brain reorganization in motor areas of the contralesional hemisphere of well recovered patients after capsular stroke. Brain 2006;129:791-808.

62 Lotze M, Markert J, Sauseng P, et al. The role of multiple contralesional motor areas for complex hand movements after internal capsular lesion. J Neurosci 2006;26:6096-102.

63 de Berker AO, Bikson M, Bestmann S. Predicting the behavioral impact of transcranial direct current stimulation: issues and limitations. Front Hum Neurosci 2013;7:613. 
64 Giacobbe V, Krebs HI, Volpe BT, et al. Transcranial direct current stimulation (tDCS) and robotic practice in chronic stroke: the dimension of timing. Neurorehabilitation 2013;33:49-56.

65 Stagg CJ, Jayaram G, Pastor D, et al. Polarity and timing-dependent effects of transcranial direct current stimulation in explicit motor learning. Neuropsychologia 2011:49:800-4.

66 Nitsche MA, Seeber A, Frommann K, et al. Modulating parameters of excitability during and after transcranial direct current stimulation of the human motor cortex. J Physiol 2005;568:291-303.
67 Zheng $X$, Alsop DC, Schlaug G. Effects of transcranial direct current stimulation (tDCS) on human regional cerebral blood flow. Neuroimage 2011;58:26-33.

68 Lang N, Siebner HR, Ward NS, et al. How does transcranial DC stimulation of the primary motor cortex alter regional neuronal activity in the human brain? Eur $\mathrm{J}$ Neurosci 2005;22:495-504.

69 Stagg CJ, Best JG, Stephenson MC, et al. Polarity-sensitive modulation of cortical neurotransmitters by transcranial stimulation. J Neurosci 2009;29:5202-6.

70 Stagg CJ, Johansen-Berg $\mathrm{H}$. Studying the effects of transcranial direct-current stimulation in stroke recovery using magnetic resonance imaging. Front Hum Neurosci 2013;7:857. 